\title{
Identification of TOMM34, which shows elevated expression in the majority of human colon cancers, as a novel drug target
}

\author{
TAKASHI SHIMOKAWA ${ }^{1}$, SATOSHI MATSUSHIMA ${ }^{1}$, TAKUYA TSUNODA $^{2}$, HIDEAKI TAHARA $^{2}$, \\ YUSUKE NAKAMURA ${ }^{1}$ and YOICHI FURUKAWA ${ }^{3}$ \\ ${ }^{1}$ Laboratory of Molecular Medicine, Human Genome Center, ${ }^{2}$ Department of Surgery and Bioengineering, and ${ }^{3}$ Promotion of \\ Genome-Based Medicine Project, Institute of Medical Science, The University of Tokyo, Tokyo 108-8639, Japan
}

Received January 31, 2006; Accepted March 23, 2006

\begin{abstract}
In an attempt to isolate potential molecular targets for diagnosis, treatment and/or prevention of colorectal cancer (CRC), we have been analyzing expression profiles of clinical samples from CRC patients using genome-wide cDNA microarray. Among the genes up-regulated frequently in colorectal tumors, we here focused on TOMM34 (34 kDatranslocase of the outer mitochondrial membrane). Immunohistochemical staining revealed significant accumulation of TOMM34 protein in CRC tissues compared with their corresponding non-cancerous mucosae. Transfection of colon cancer HCT116 cells with short-interfering RNA (siRNA) specific to TOMM34 effectively suppressed its expression and drastically inhibited cell growth. These findings suggest that TOMM34 is involved in the growth of cancer cells, and may contribute to the development of novel anticancer drugs and/or diagnosis for CRC.
\end{abstract}

\section{Introduction}

Colorectal cancer is one of the most common causes of cancerdeath worldwide. Despite various advances in the diagnosis and treatment of CRC in recent decades, many patients with advanced CRC succumb to the disease. To improve prognoses, development of sensitive and specific diagnostic biomarkers for the detection of early-stage carcinomas and that of more effective and less harmful therapeutic drugs are desired. To attain this end, it is requisite to better understand the molecular mechanisms of colorectal carcinogenesis. Molecular studies have revealed that colorectal carcinogenesis involves an accumulation of genetic alternations that include genetic changes in tumor suppressor genes and/or oncogenes including

Correspondence to: Dr Yoichi Furukawa, Promotion of GenomeBased Medicine Project, Institute of Medical Science, The University of Tokyo, 4-6-1 Shirokanedai, Minato-ku, Tokyo 108-8639, Japan

E-mail: furukawa@ims.u-tokyo.ac.jp

Key words: TOMM34, colon cancer, molecular target
$A P C, p 53, \beta$-catenin and $K$-ras (1-4). In addition, epigenetic events such as altered methylation (5) and loss of imprinting (6), and/or deregulated transcriptional control by genetic changes or other unknown mechanism(s) are involved in the genesis of colorectal tumors. Among the genes involved in carcinogenesis, we can expect that inhibition of gene products essential for proliferation and/or survival of cancer cells will result in their growth inhibition or cell death. Therefore, molecules that exert oncogenic activity and are specifically expressed in cancer cells represent promising targets for developing novel anti-cancer drugs.

Mitochondrial translocation machinery is a fundamental mechanism for normal mitochondrial function. It is achieved by several components including translocase of the outer membrane of mitochondria (Tom) and that of the inner membrane (Tim). Among the Tom components, Tom 20 and Tom22, and Tom 37 and Tom70, form heterodimeric receptor complexes that recognize target protein to translocate. Human TOMM34 was identified from human EST and cDNA databases and predicted to encode a protein containing degenerated tetratricopeptide repeat (TPR) motifs. The predicted protein shares sequence homology in a 62-residue motif with known yeast Tom70 family of mitochondrial receptors (7). In addition, antibodies against Tom34 inhibited translocation of preprotein into mitochondria (8). These data indicated that TOMM34 plays a role in mitochondrial import of preprotein. Investigations revealed that the $\mathrm{NH} 2$-terminal region of Tom34 is essential for the protein import, and that it interacts with the mature portion of some preproteins (8). In addition, recent studies proposed a possible function of Tom to keep preproteins in unfolded importable protein conformation (9). However, the precise role of TOMM34 remains unresolved.

To clarify the molecular mechanisms in colorectal carcinogenesis and find novel therapeutic targets for CRC, we previously performed global gene-expression profile analysis using a cDNA microarray consisting of 23040 genes (10). In this study, we report that human TOMM34 is frequently up-regulated in CRCs, and that it is expressed abundantly in the testis and ovary, at low levels in the prostate, spleen, and colon, and barely in the other 11 normal adult tissues examined. Since suppression of TOMM34 by siRNA markedly reduced the growth of colon cancer cells, the gene product may be a potential therapeutic target for human tumors as well as a useful diagnostic maker. 


\section{Materials and methods}

Cell lines and clinical materials. Human colon cancer cell lines HCT116 DLD1, HCT15, SW480, monkey fibroblast line COS7, and murine fibroblast line NIH3T3 were obtained from the American Type Culture Collection (ATCC, Rockville, MD). A human colon cancer cell line, SNU C4, was obtained from Korean cell line bank (KCLB, Seoul, Korea). LS174T and RKO cells were gifts from Drs H. Fukamachi (Tokyo Medical and Dental University, Japan) and from C.C. Harris (NCI, Washington DC), respectively. All cells were cultured as monolayers in appropriate media; McCoy's 5A (Invitrogen, Carlsbad, CA) for HCT116, Leibovitz's L-15 (Invitrogen) for SW480, RPMI-1640 (Sigma-Aldrich, St. Louis, MO) for SNU C4, DLD1, HCT15, and HCT116, Eagle's minimum essential medium (Sigma) for LS174T, and Dulbecco's modified Eagle's medium (Sigma) for RKO, COS7, and NIH3T3, supplemented with $10 \%$ fetal bovine serum (Cansera, Ontario, Canada) and $1 \%$ antibiotic/antimycotic solution (Sigma). Cells were maintained at $37^{\circ} \mathrm{C}$ in an atmosphere of humidified air with (DLD1, HCT15, HCT116, SNUC4, RKO, LS174T, COS7, and NIH3T3) or without (SW480) $5 \% \mathrm{CO}_{2}$. Cancerous tissues and corresponding non-cancerous mucosae were excised from 20 patients during surgery, after informed consent had been obtained.

Semi-quantitative RT-PCR. Total RNA was extracted from cultured cells or clinical tissues using TRIzol reagent (Invitrogen) according to the manufacturer's protocol. Extracted RNA was treated with DNaseI (Roche Diagnostics, Mannheim, Germany) and reverse transcribed to singlestranded cDNAs using oligo(dT) $)_{12-18}$ primers and Superscript II reverse transcriptase (Invitrogen). We prepared appropriate dilutions of each single-stranded cDNA for subsequent PCR amplification by monitoring the $G A P D H$ gene as a quantitative control. The primer sequences used were 5'-ACAACAGCCT CAAGATCATCAG-3' and 5'-GGTCCACCACTGACACGT TG-3' for GAPDH; 5'-TGGTATAAACCTAAGGCCCTG AT-3' and 5'-TAAACAGCTTAGGTGCCTCTCTG-3' for TOMM34. All of the amplification reactions were preceded by initial denaturation at $94^{\circ} \mathrm{C}$ for $2 \mathrm{~min}$, followed by 18 (for GAPDH) or 29 cycles (for TOMM34) of amplification at $94^{\circ} \mathrm{C}$ for $30 \mathrm{sec}, 60^{\circ} \mathrm{C}$ for $30 \mathrm{sec}$, and $72^{\circ} \mathrm{C}$ for $30 \mathrm{sec}$ on a GeneAmp PCR system 9700 (PE Applied Biosystems, Foster, CA).

Northern blotting. Human multiple-tissue blots (BD Bioscience, Palo Alto, CA) were hybridized with a ${ }^{32} \mathrm{P}-$ labeled PCR product of TOMM34. The probe was prepared by RT-PCR using a set of primers, 5'-GAACGTGAAGGCA TTCTACAGA-3' and 5'-TAAACAGCTTAGGTGCCTCTC TG-3', and subsequent random-oligonucleotide labeling with ${ }^{32} \mathrm{P}-\mathrm{dCTP}$ using a Mega Label kit (Amersham Biosciences, Buckinghamshire, UK). Prehybridization, hybridization, and washing were performed according to the supplier's recommendations. The blots were autoradiographed with intensifying screens at $-80^{\circ} \mathrm{C}$ for 10 days.

Preparation of polyclonal antibody against TOMM34. The entire coding region of TOMM34 was amplified using a set of primers, 5'-CATAAGCTTGCATGGCCCCCAAATT CCCA-3' and 5'-GTTAAGCTTTTAGTGTAGGTTCT-3', and subsequently cloned into an appropriate cloning site of pET28 vector (Novagen, Madison, WI) to generate plasmids expressing His-tagged TOMM34 protein. The recombinant protein was expressed in Escherichia coli, BL21-CodonPlus (DE3)-RIL strain (Stratagene, La Jolla, CA), and purified using TALON Superflow Metal Affinity Resin (BD Bioscience) according to the manufacturer's protocol. The protein was inoculated into rabbits, and the immunized sera were purified on affinity columns according to the standard method. Western blot analysis was carried out using proteins extracted from the cells using $0.1 \%$ RIPA like buffer containing $50 \mathrm{mM}$ Tris- $\mathrm{HCl}$ (pH 7.5), $250 \mathrm{mM} \mathrm{NaCl}, 0.1 \%$ SDS, and 0.5\% NP40 with Protease Inhibitor Cocktail Set III (CALBIOCHEM, La Jolla, CA).

Immunohistochemistry. Immunohistochemical staining was performed using affinity-purified polyclonal antibody against human TOMM34. The paraffin-embedded tissue sections were subjected to the SAB-PO peroxidase immunostaining system (Nichirei, Tokyo, Japan), after antigens were retrieved from deparaffinized and re-hydrated tissues by pre-treating the slides in $0.01 \mathrm{M}$ citrate buffer $(\mathrm{pH} 6.0)$ at $108^{\circ} \mathrm{C}$ for $10 \mathrm{~min}$ by autoclave.

Construction of psiU6BX expressing siRNAs to TOMM34. Plasmids expressing siRNAs were prepared by cloning double-stranded oligonucleotides into the $B b s I$ site in the psiU6BX vector as described previously (11). The sequences of paired oligonucleotides were 5'-CACCGAAAGTGTTCTC TACTCCATTCAAGAGATGGAGTAGAGAACACTTTC-3' and 5'-AAAAGAAAGTGTTCTCTACTCCATCTCTTGAA TGGAGTAGAGAACACTTTC-3' for siD; 5'-CACCGG ATGGAAACTGCAGAGACTTCAAGAGAGTCTCTGCA GTTTCCATCC-3' and 5'-AAAAGGATGGAAACTGCAGA GACTCTCTTGAAGTCTCTGCAGTTTCCATCC-3' for siE; 5'-CACCTAAAGTCTTATCTACTCCATTCAAGAGA TGGAGTAGATAAGACTTTA-3' and 5'-AAAATAAAGTC TTATCTACTCCATCTCTTGAATGGAGTAGATAAGAC TTTA-3' for siDmm; and 5'-CACCGATCTCTAGTACTAG TCCATTCAAGAGATGGACTAGTACTAGAGATC-3' and 5'-AAAAGATCTCTAGTACTAGTCCATCTCTTGAATGG ACTAGTACTAGAGATC-3' for siDscr. TOMM34-siDmm and TOMM34-siDscr expressed mutant siRNAs containing either three nucleotides substitution or scrambled sequence of TOMM34-siD, respectively. After phosphorylation with T4polynucleotide kinase, the paired oligonucleotides were boiled for $5 \mathrm{~min}$, and were subsequently annealed to produce double-stranded oligonucleotides by cooling slowly. A control plasmid, psiU6BX-EGFP was prepared using 5'-CACCGAA GCAGCACGACTTCTTCTTCAAGAGAGAAGAAGTCGT GCTGCTTC-3' and 5'-AAAAGAAGCAGCACGACTTC TTCTCTCTTGAAGAAGAAGTCGTGCTGCTTC-3'. To examine the effect of TOMM34-siRNAs, Western blot analysis was carried out using anti-TOMM34 antibody five days after transfection with the plasmids.

Colony-formation assay. HCT116 cells plated on 10-cm dishes $\left(4 \times 10^{5}\right.$ cells/dish) were transiently transfected with plasmids expressing TOMM34-siRNAs using FuGENE 6 reagent (Roche Diagnostics), and maintained in a medium containing $10 \%$ fetal bovine serum with $800 \mu \mathrm{g} / \mathrm{ml}$ Geneticin for two 


\section{a}

\begin{tabular}{|c|c|c|c|c|c|c|c|c|c|c|c|c|c|c|c|c|}
\hline & $\mathrm{N}$ & $\mathrm{T}$ & $\underline{\mathrm{N}}$ & $\mathrm{T}$ & $\mathrm{N}$ & $\mathrm{T}$ & $\underline{\mathrm{N}}$ & $T$ & $\mathrm{~N}$ & $\mathrm{~T}$ & $\mathrm{~N}$ & $T$ & $\mathrm{~N}$ & $T$ & $\mathrm{~N}$ & $T$ \\
\hline томмз4 & 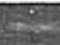 & 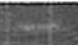 & & & & 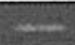 & $=$ & - & $=$ & - & & - & & & & $=$ \\
\hline & $=$ & - & - & $\Rightarrow$ & $\longrightarrow$ & - & - & - & - & - & - & - & - & $=$ & - & - \\
\hline & $\mathrm{N}$ & $\mathrm{T}$ & $\mathrm{N}$ & $T$ & $\mathrm{~N}$ & $T$ & $\underline{\mathrm{N}}$ & $T$ & $\mathrm{~N}$ & $\mathrm{~T}$ & $\mathrm{~N}$ & T & $\mathrm{N}$ & $\mathrm{T}$ & $\mathrm{N}$ & $T$ \\
\hline DMM34 & $=$ & - & & s. & tous & - & & $\sin$ & 5 & - & & 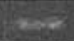 & & 5 & & 5 \\
\hline $4 P D$ & & & & & & & & & & - & & - & 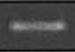 & 2 & 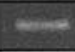 & - \\
\hline
\end{tabular}

\begin{tabular}{|c|c|c|c|c|c|c|c|c|}
\hline & & & $\mathrm{N}$ & $\mathrm{T}$ & $\mathrm{N}$ & $T$ & $\underline{N}$ & $\mathrm{~T}$ \\
\hline$M$ & & - & & $\rightarrow$ & & - & & $\rightarrow$ \\
\hline & & - & & - & & & & \\
\hline
\end{tabular}

b

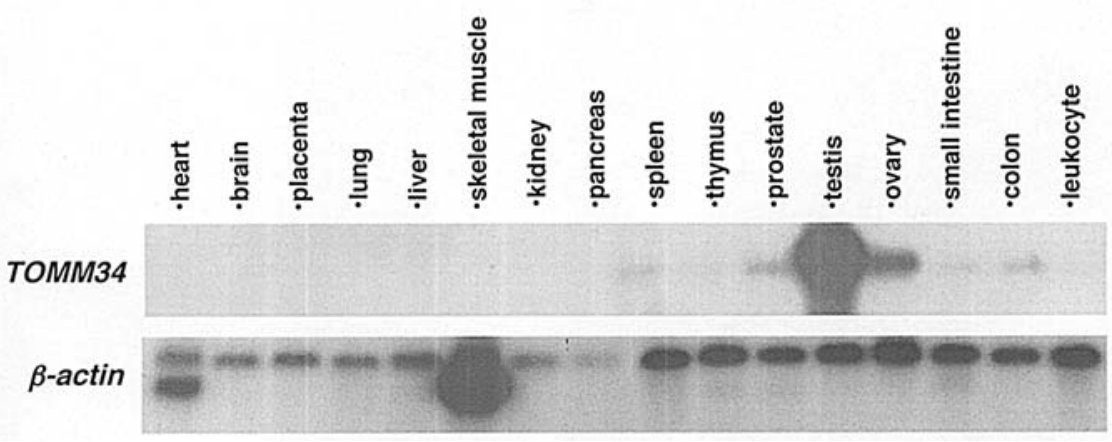

Figure 1. (a) Semi-quantitative RT-PCR analysis of TOMM34 in 20 colon cancer tissues and their corresponding non-cancerous mucosa. T, tumor tissue; N, normal tissue. Expression of GAPDH served as an internal control. (b) Multiple-tissue Northern blot analysis of TOMM34. The transcript of TOMM34 is approximately $2.0 \mathrm{~kb}$ by size.

a

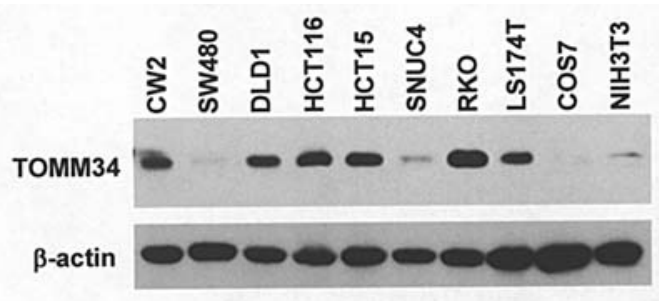

b
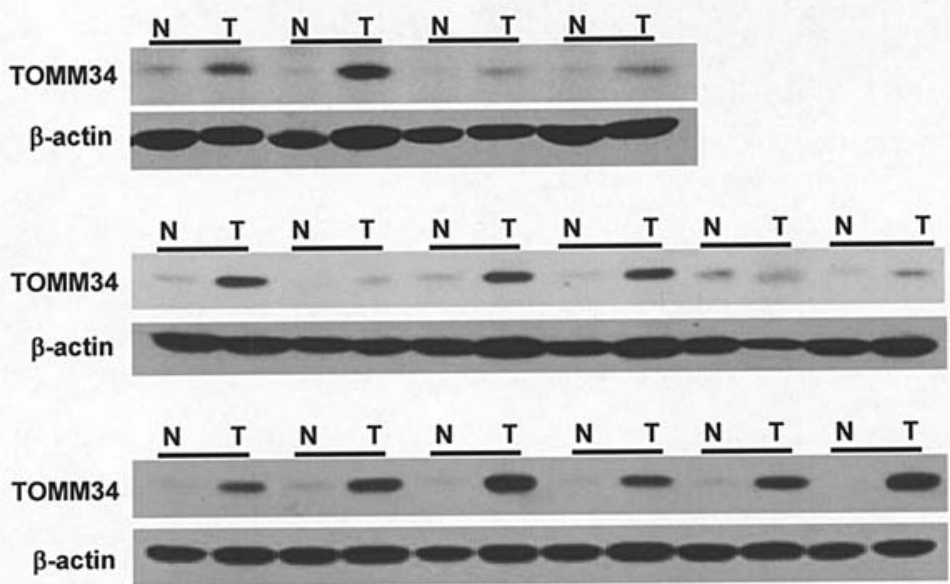

Figure 2. (a) Expression of TOMM34 protein in colon cancer cell lines. Expression of B-actin served as an internal control. (b) Western blot analysis of TOMM34 in 16 colon cancer tissues and their corresponding non-cancerous mucosa. $\mathrm{T}$, tumor tissue; $\mathrm{N}$, normal tissue.

weeks. The surviving cells were fixed with $100 \%$ methanol and stained with Giemsa solution. In another experiment, the viable cells were measured using a cell-counting kit (DOJINDO, Kumamoto, Japan). 

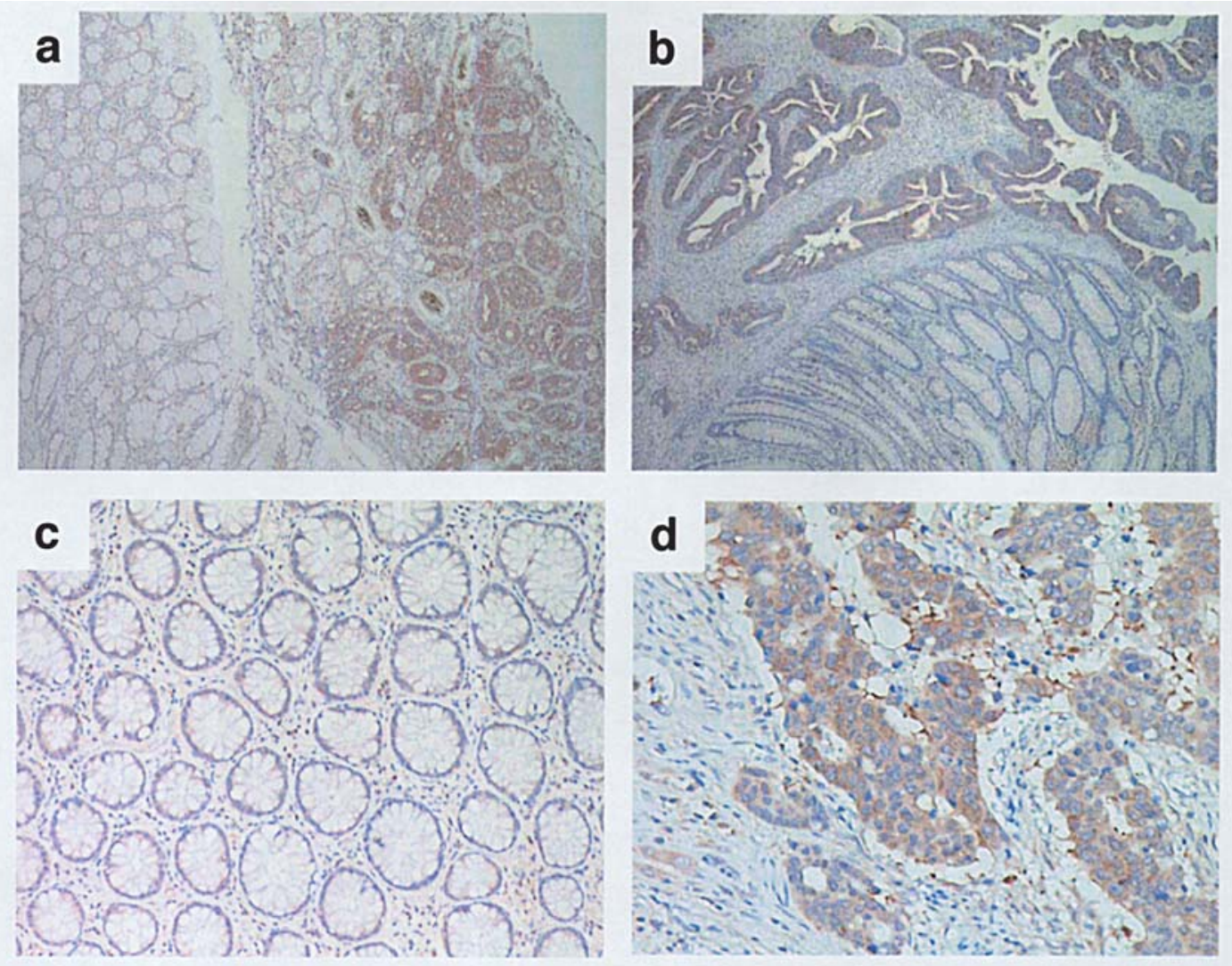

Figure 3. Immunohistochemical staining of TOMM34 in colon cancer tissues. Representative images of the staining in cancerous and non-cancerous cells (a, b and d). A representative image of normal mucasa (c). Magnification, x40 (a and b), x200 (c and d).

a

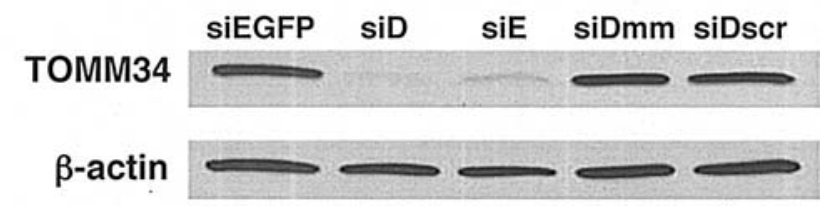

b

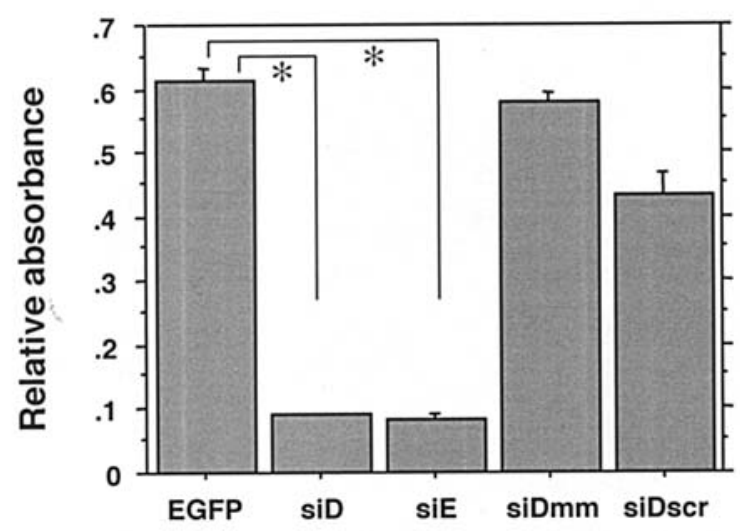

Figure 4. (a) Effect of siRNAs on the expression of TOMM34 in HCT116 cells. Expression of TOMM34 was analyzed by Western blot analysis. Expression of $\beta$-actin served as an internal control. EGFP-specific siRNA (siEGFP) was prepared for a negative control, and siDmm and siDscr are two mutant forms of TOMM34-siD. (b) Effect of TOMM34-siRNAs on the growth of HCT116 cells. Viability of HCT116 cells in response to EGFPsiRNA or TOMM34-siRNAs was measured by MTT assay in triplicate. Error bars, SD; *significant difference $(\mathrm{P}<0.0001)$ determined by Scheff's $\mathrm{F}$ test.
Statistical analysis. Statistical significance was analyzed by ANOVA with Scheff's F test, using StatView software (SAS Institute, Cary, NC).

\section{Results}

Elevated expression of TOMM34 in CRCs. In our earlier study, we identified a number of genes whose expression levels were frequently altered in colon tumors by the genomewide expression profile analysis of 11 CRCs and 9 adenomas of the colon using a cDNA microarray consisting of 23040 genes (10). Among the genes whose expression levels were frequently up-regulated in the 11 carcinomas, we focused in this study on a gene with in-house identification number D3124, which corresponded to TOMM34 (34-kDa translocase of the outer mitochondrial membrane). Subsequent semiquantitative RT-PCR analysis revealed its enhanced expression in 16 of 20 CRC clinical samples examined (Fig. 1a). In addition, TOMM34 was also up-regulated in all 6 hepatocellular carcinomas, 5 of 20 lung cancers, 8 of 11 bladder cancers, 4 of 28 acute myeloid leukemias, and 10 of 51 soft tissue sarcomas in our cDNA microarray data (data not shown). To investigate its expression in human normal tissues, we performed multiple-tissue Northern blot analysis using TOMM34 cDNA as a probe. As a result, the analysis revealed a transcript of approximately $2.0 \mathrm{~kb}$ by size, which was abundantly expressed in the testis and ovary, and weakly in the prostate, spleen, and colon, but no bands were detected in any of the other 11 tissues examined (Fig. 1b). 
Accumulated TOMM34 protein in colorectal cancer cell lines and CRC tissues. We prepared anti-TOMM34 polyclonal antibody and examined expression of TOMM34 protein in eight CRC cell lines and 16 CRC tissues. Immunoblot analysis detected a 34-kDa band of TOMM34 that was abundantly expressed in all CRC cell lines examined. On the other hand, low levels of expression were shown in NIH3T3 and COS7, two non-cancerous cell lines (Fig. 2a). Western blot analysis using 16 CRCs and corresponding non-cancerous mucosal tissues demonstrated its enhanced expression in 15 of the 16 tumors compared to the normal mucosa (Fig. 2b). To examine the subcellular localization of TOMM34, we carried out immunocytochemical staining using HCT116 and RKO cells with the anti-TOMM34 antibody, which showed cytoplasmic localization of the protein (data not shown). In addition, we performed immunohistochemical staining using 12 paraffin-embedded colon cancer tissues. In 11 of the 12 tumors, TOMM34 was strongly stained in the cytoplasms of cancerous cells, but it was barely stained in non-cancerous mucosa (Fig. 3).

Effect of TOMM34-siRNAs on growth of colon cancer cells. To elucidate the role of TOMM34 in cancer cells, we prepared two forms of plasmids expressing siRNAs designated to suppress TOMM34 (psiU6BX-TOMM34-siD, and -siE) and a control plasmid (psiU6BX-siEGFP). We transfected HCT116 cells with psiU6BX-TOMM34-siD, psiU6BX-TOMM34-siE, or psiU6BX-siEGFP, and examined their effect on the expression of TOMM34. Western blot analysis of extracts from transfected cells revealed that psiU6BX-TOMM34-siD and psiU6BX-TOMM34-siE significantly suppressed TOMM34 expression in the transfected cells compared to psiU6BX-siEGFP (Fig. 4a). We additionally constructed two forms of mutant plasmids containing either a three-nucleotide substitution or a scrambled sequence of TOMM34-siD (psiU6BX-TOMM34-siDmm or psiU6BX-TOMM34-siDscr, respectively). Expectedly, both forms of mutant plasmids failed to suppress TOMM34 expression, indicating sequence specific inhibition of TOMM34 by psiU6BX-TOMM34-siD (Fig. 4a). We transfected plasmids co-expressing the neomycinresistance gene into HCT116 cells, and cultured them with an appropriate concentration of geneticin for 13 days. In accordance with reduced expression of TOMM34, both psiU6BXTOMM34-siD and psiU6BX-TOMM34-siE markedly retarded the growth of transfected cells compared to psiU6BX-siEGFP. Concordant with the unchanged expression of TOMM34, psiU6BX-TOMM34-siDmm or psiU6BX-TOMM34-siDscr did not affect the number of viable cells compared with the control plasmid (Fig. 4b). These data indicated that expression of TOMM34 is associated with growth of cancer cells.

\section{Discussion}

In this study, we focused on TOMM34, which is reported to be involved in mitochondrial import of preprotein, because it was frequently up-regulated in CRCs in our microarray data. Mitochondrial activity is enhanced in cancer cells because of augmented metabolism, which is one of the common features of highly proliferating cells. Consistent with this view, the microarray data revealed that TOMM34 expression is enhanced in a wide range of human cancers. Since energy production is essential for survival of cancer cells, suppression of mitochondrial activity should lead to their growth retardation or death. The growth suppression by siRNA to TOMM34 in cancer cells supports this notion.

Human TOMM34 was identified by screening for a degenerate tetratricopeptide repeat (TPR) motif that had been found in many Tom proteins. TOMM34 contains six TPR motifs, which consist of 34 amino-acid repeat sequences, and are involved in protein-protein interaction. Proteins containing this motif play a role in diverse cellular pathways; Cdc16p, Cdc23p, and Cdc27p are components of the cyclosome/APC multicomplex, and are required for cell cycle progression through mitosis (12); FKBP4 and FKBP5, two immunophilins, are mediators of the immunoreaction $(13,14)$. TOMM34 was initially predicted to play a role in mitochondrial protein translocation as a mitochondrial outer membrane protein. However, its cytoplasmic localization and its interaction with heat shock protein 90 suggested a role in maturation of newly synthesized proteins $(15,16)$. The yeast two-hybrid screening system has shown that TOMM34 interacts in vitro with valosin-containing protein (VCP), an AAA (ATPases associated with a variety of cellular activities) family member, and ATPase, $\mathrm{H}+$ transporting, lysosomal $34 \mathrm{kDa}, \mathrm{V} 1$ subunit $\mathrm{D}$ (ATP6V1D or ATP6M) (16). VCP has been shown to be essential for normal cellular function; cell cycle, homotypic membrane fusion, nuclear envelope reconstruction, postmitotic Golgi reassembly, DNA damage response, suppressor of apoptosis, and ubiquitin-dependent protein degradation $(17,18)$. ATP6V1D (or ATP6M) was isolated as a gene encoding D subunit of vacuolar proton pump ATPase (19). Notably, both VCP and ATP6V1D are essential for cell division (20). Therefore, growth retardation of cancer cells by TOMM34 siRNAs may result from abrogated mitotic progression through inhibition of VCP and/or ATP6V1D. Since a region containing the TPR motif between codons 227 and 260 is responsible for the interaction (16), molecules that interfere with the binding may disrupt the function of TOMM34. Additionally, TOMM34 has ATPase activity, although the activity is weaker than VCP or ATP6V1D (16). Therefore, inhibitors of the ATPase activity may be an alternative strategy to inhibit the function of TOMM34.

It is of note that TOMM34 null mice were fertile and developed normally, suggesting that it is dispensable for development and growth under an environmentally normal condition (21). TOMM34 is expressed abundantly in the testis and ovary, and expressed at low levels in prostate, spleen, and colon, but rarely expressed in other normal adult tissues including heart, lung, kidney and liver. Therefore, it is unlikely that the inhibition of TOMM34 will bring severe adverse effects on those vital tissues. From this point of view, inhibitors of TOMM34 should be a good anticancer drug with no or minimum adverse effects. In addition, suppression of TOMM34 resulted in the inhibition of cell growth of cancer cells, suggesting that TOMM34 plays an essential role in their growth. These findings imply that TOMM34 is a promising target for immunotherapy and/or development of anticancer drugs.

\section{Acknowledgements}

We thank R. Hamamoto, M. Sakai, K. Obama, K. Ura, T. Kobayashi, and M. Tsuge for their helpful discussions, and 
T. Nagao for excellent technical assistance. This work was supported in part by a Grant-in-Aid for Scientific Research from the Ministry of Education, Culture, Sports, Science and Technology, Japan.

\section{References}

1. Nishisho I, Nakamura Y, Miyoshi Y, Miki Y, Ando H, Horii A, Koyama K, Utsunomiya J, Baba S and Hedge P: Mutation of chromosome 5q21 genes in FAP and colorectal cancer patients. Science 253: 665-669, 1991.

2. Baker SJ, Fearon ER, Nigro JM, Hamilton SR, Preisinger AC, Jessup JM, van Tuinen P, Ledbetter DH, Baker DF, Nakamura Y, White R and Vogelstein B: Chromosome 17 deletions and p53 gene mutations in colorectal carcinomas. Science 244: 217-221, 1989.

3. Morin PJ, Sparks AB, Korinekn V, Barker N, Clevers H, Vogelstein B and Kinzler KW: Activation of beta-catenin-Tcf signaling in colon cancer by mutations in beta-catenin or APC. Science 275: 1787-1790, 1997.

4. Forrester K, Almoguera C, Han K, Grizzle WE and Perucho M: Detection of high incidence of K-ras oncogenes during human colon tumorigenesis. Nature 327: 298-303, 1987.

5. Jones PA and Laird PW: Cancer epigenetics comes of age. Nat Genet 21: 163-167, 1999.

6. Cui H, Horon IL, Ohlsson R, Hamilton SR and Feinberg AP: Loss of imprinting in normal tissue of colorectal cancer patients with microsatellite instability. Nat Med 4: 1276-1280, 1998.

7. Nuttall SD, Hanson BJ, Mori M and Hoogenraad NJ: hTom34: a novel translocase for the import of proteins into human mitochondria. DNA Cell Biol 16: 1067-1074, 1997.

8. Chewawiwat N, Yano M, Terada M, Hoogenraad NJ and Mori M: Characterization of the novel mitochondrial protein import component, Tom34, in mammalian cells. J Biochem 125: 721-727, 1999.

9. Mukhopadhyay A, Avramova LV and Weiner H: Tom34 unlike Tom20 does not interact with the leader sequences of mitochondrial precursor proteins. Arch Biochem Biophys 400: 97-104, 2002.

10. Lin YM, Furukawa Y, Tsunoda T, Yue CT, Yang KC and Nakamura Y: Molecular diagnosis of colorectal tumors by expression profiles of 50 genes expressed differentially in adenomas and carcinomas. Oncogene 21: 4120-4128, 2002.
11 Shimokawa T, Furukawa Y, Sakai M, Li M, Miwa N, Lin YM and Nakamura Y: Involvement of the FGF18 gene in colorectal carcinogenesis, as a novel downstream target of the betacatenin/T-cell factor complex. Cancer Res 63: 6116-6120, 2003.

12. Lamb JR, Michaud WA, Sikorski RS and Hieter PA: Cdc16p, Cdc23p and Cdc27p form a complex essential for mitosis. EMBO J 13: 4321-4328, 1994.

13. Radanyi C, Chambraud B and Baulieu EE: The ability of the immunophilin FKBP59-HBI to interact with the $90-\mathrm{kDa}$ heat shock protein is encoded by its tetratricopeptide repeat domain. Proc Natl Acad Sci USA 91: 11197-11201, 1994.

14. Owens-Grillo JK, Czar MJ, Hutchinson KA, Hoffmann K, Perdew GH and Pratt WB: A model of protein targeting mediated by immunophilins and other proteins that bind to hsp90 via tetratricopeptide repeat domains. J Biol Chem 271: 13468-13475, 1996.

15. Young JC, Obermann WM and Hartl FU: Specific binding of tetratricopeptide repeat proteins to the C-terminal $12-\mathrm{kDa}$ domain of hsp90. J Biol Chem 273: 18007-18010, 1998.

16. Yang CS and Weiner H: Yeast two-hybrid screening identifies binding partners of human Tom34 that have ATPase activity and form a complex with Tom34 in the cytosol. Arch Biochem Biophys 400: 105-110, 2002.

17. Dai RM and Li CC: Valosin-containing protein is a multiprotein chain-targeting factor required in ubiquitin-proteasome degradation. Nat Cell Biol 3: 740-744, 2001.

18. Watts GD, Wymer J, Kovach MJ, Mehta SG, Mumm S, Darvish D, Pestronk A, Whyte MP and Kimonis VE: Inclusion body myopathy associated with Paget disease of bone and frontotemporal dementia is caused by mutant valosin-containing protein. Nat Genet 36: 377-381, 2004.

19. Kennell JA, Richards NW, Schaner PE and Gumucio DL: cDNA cloning, chromosomal localization and evolutionary analysis of mouse vacuolar ATPase subunit D, Atp6m. Cytogenet Cell Genet 92: 337-341, 2001.

20. Kittler R, Putz G, Pelletier L, Poser I, Heninger AK, Drechsel D, Fischer S, Konstantinova I, Habermann B, Grabner H, Yaspo ML, Himmelbauer H, Korn B, Neugebauer K, Pisabarro MT and Buchholz F: An endoribonuclease-prepared siRNA screen in human cells identifies genes essential for cell division. Nature 432: 1036-1040, 2004.

21. Terada K, Ueno S, Yomogida K, Imai T, Kiyonari H, Takeda N, Yano M, Abe S, Aizawa S and Mori M: Expression of Tom34 splicing isoforms in mouse testis and knockout of Tom34 in mice. J Biochem 133: 625-631, 2003. 